\title{
NEW YEAST SPECIES AND VARIETIES HITHERTO DESCRIBED IN JAPAN
}

\author{
SHOJI GOTO, TAKASHI NAKASE,* ISAO BANNO,** \\ AND TAKESHI TSUCHIYA*** \\ Research Institute of Fermentation, Yamanashi University, Kofu, \\ *Central Research Laboratories, Ajinomoto Co., Inc., Kawasaki, \\ **Institute for Fermentation, Osaka, and ${ }^{* * *}$ School of \\ Medicine, Juntendo University, Tokyo
}

(Received April 1, 1975)

In Japan, studies on the yeast started in the field of brewing and fermentation, and of botany, zoology, and medicine about the end of the 19th century. A number of new yeast species and varieties have since been reported in these fields. A considerable number of these yeast species were reclassified by workers of the Dutch school (1-5), but others still remained unstudied on account of difficult understanding of Japanese language for foreign people, incomplete descriptions, and lack of authentic cultures.

The present authors have conducted a survey to verify the original descriptions, authentic cultures and their maintenance, and the synonyms of the new yeast species and varieties reported in Japan until 1971.

As a result, 236 new species and 76 new varieties are listed. They belong to 34 genera as shown in Table 1. Saccharomyces sake Yabe, which is used for brewing of Saké, was first reported in Japan in 1897. Several new genera have been described to date, and Wickerhamia Soneda 1960 and Rhodosporidium Banno 1967 have been widely accepted by yeast taxonomists.

Of these new 236 species 193 authentic cultures, and of 76 varieties 51 authentic cultures have been deposited in various culture collections. These new taxa have been published in 34 Japanese journals and 4 foreign ones.

In spite of this extensive survey, however, there may be papers other than those listed here. The present authors would appreciate any information from microbiologists who have interest in this field.

*** Present address: Department of Serology, Kanagawa Prefectural College, Yokohama. 
This research survey was supported in part by a Grant-in-Aid for Scientific Research (No. 0705, 1971) from the Japanese Ministry of Education.

Table 1. Genera to which new species and varieties belong,

\begin{tabular}{|c|c|c|c|c|c|}
\hline \multirow{2}{*}{ Genus } & \multicolumn{2}{|c|}{ Number of } & \multirow{2}{*}{ Genus } & \multicolumn{2}{|c|}{ Number of } \\
\hline & Species & Variety & & Species & Variety \\
\hline Brettanomyces & 1 & & Pseudomonilia & 2 & \\
\hline Bullera & 1 & & Pseudomycoderma & 2 & \\
\hline Candida & 34 & 10 & Rhodosporidium & 1 & \\
\hline Cryptococcus & 2 & 2 & Rhodotorula & 4 & 4 \\
\hline Debaryomyces & 21 & 3 & Saccharomyces & 26 & 16 \\
\hline Endomyces & 2 & & Sarcinomyces & 1 & \\
\hline Endomycopsis & 4 & & Schizoblastosporion & 1 & \\
\hline Eutorulopsis & 2 & & Schizosaccharomyces & 6 & 6 \\
\hline Hansenula & 4 & 4 & Sporobolomyces & 8 & \\
\hline Kloeckera & 3 & & Torula & 26 & 3 \\
\hline Myceloblastanon & 6 & & Torulaspora & 2 & \\
\hline Mycoderma & 1 & & Torulopsis & 26 & 9 \\
\hline Mycotorula & 2 & 1 & Trichosporon & 7 & 2 \\
\hline Naganishia & 1 & & Wickerhamia & 1 & \\
\hline Oospora & 2 & & Zygopicha & 2 & 3 \\
\hline Pichia & 15 & & Zygosaccharomyces & 17 & 15 \\
\hline Pseudohansenula & 2 & & Zygosaccharomycodes & 1 & \\
\hline
\end{tabular}

\section{REFERENCES}

1) N. M. Stelling-Dekker. Die sporogenen Hefen. Verh. K. Akad. Wet. Amsterdam, Afd. Natuurkd., Sec. II, 28, 1 (1931).

2) J. LodDer. Die anaskosporogenen Hefen. I Hälfle. Verh. K. Akad. Wet. Amsterdam, Afd. Natuurkd., Sec. II, 32, 1 (1934).

3) H. A. DidDens and J. LoDDER. "Die anaskosporogenen Hefen," II Hälfte, North Holland Publ., Amsterdam (1942).

4) J. Lodder and N. J. W. Kreger-van RiJ. "The Yeasts, A Taxonomic Study, "NorthHolland Publ. Co., Amsterdam (1952).

5) J. Lodder (ed.), "The Yeasts, A Taxonomic Study," 2nd Ed., North-Holland Publ. Co., Amsterdam-London (1970).

Yeast names are alphabetically arranged and the numbers indicate the following items:

1. Number of authentic culture.

2. Accession number of culture collection in which the authentic culture has been deposited.

3. Original literature.

4. Synonym according to "The Yeasts", 2nd ed. (1970) unless otherwise stated. 


\section{Abbreviations of Culture Collections}

AKU Faculty of Agriculture, Kyoto University, Kyoto.

ATCC American Type Culture Collection, U.S.A.

CBS Centraalbureau voor Schimmelcultures, The Netherlands.

FERM Fermentation Research Institute, Agency of Industrial Science and Technology, Chiba.

IAM Institute of Applied Microbiology, University of Tokyo, Tokyo.

IFO Institute for Fermentation, Osaka.

NI Nagao Institute, Tokyo.

OUT Faculty of Engineering, Osaka University, Suita.

RIFY Research Institute of Fermentation, Yamanashi University, Kofu.

Brettanomyces petrophilum Iguchi, Takeda et Senoo

1. 200 2. ATCC 20224 3. T. Iguchi, I. Takeda and S. Senoo: Japanese Patent 65-20666 (1965), in Japanese.

Bullera stramina Yamazaki et Fujii

1. Bu-A 3. I. Yamazaki and K. Fujii: J. Agr. Chem. Soc. Japan, 24, 11 (1950), in Japanese.

Candida australis Goto, Sugiyama et lizuka

1. AY 14 2. RIFY AY 14 3. S. Goto, J. Sugiyama and H. Iizuka: $M y-$ cologia, 61, 748 (1969), in English, with Latin description.

Candida boleticola Nakase

$\begin{array}{llll}\text { 1. AJ } 4703 & \text { 2. IFO } 1570 & \text { 3. T. Nakase: J. Gen. Appl. Microbiol., 17, } 469\end{array}$ (1971), in English, with Latin description.

Candida buinensis Soneda et Uchida

1. No. 65 A 3. M. Soneda and S. Uchida: Bull. Nat. Sci. Mus., Tokyo, 14, 448 (1971), in English, with Latin description.

Candida butyri Nakase

$\begin{array}{llll}\text { 1. AJ } 4668 & \text { 2. IFO } 1571 & \text { 3. T. Nakase: J. Gen. Appl. Microbiol., 17, } 469\end{array}$

(1971), in English, with Latin description.

Candida citrea Nakase

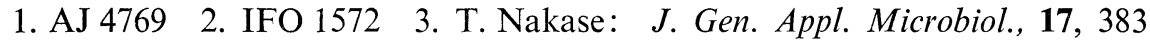

(1971), in English, with Latin description.

Candida cloacae Komagata, Nakase et Katsuya

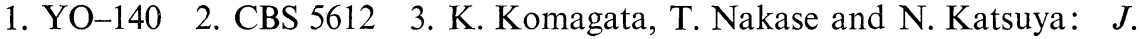

Gen. Appl. Microbiol., 10, 323 (1964), in English, with Latin description.

4. Candida sake (Saito et Ota) van Uden et Buckley.

Candida corniculata Kuraishi

1. B 84 3. H. Kuraishi: Sci. Rep. Tohoku Univ. Ser. IV, 24, 59 (1958), in

English, with Latin description.

Candida curiosa Komagata et Nakase

1. P-6 2. IFO 1336 3. K. Komagata and T. Nakase: J. Gen. Appl. 
Microbiol., 11, 255 (1965), in English, with Latin description.

Candida cylindracea Yamada et Machida

1. No. 5 3. K. Yamada and H. Machida: J. Agr. Chem. Soc. Japan, 36, 858 (1962), in Japanese.

Candida fabianii Kodama, Kyono, Iida et Onoyama

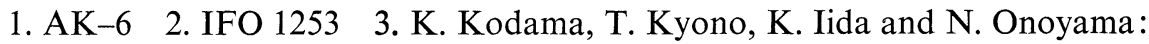

J. Ferment. Technol., 42, 739 (1964), in Japanese, with Latin description.

4. Hansenula favianii Wickerham.

Candida fibrae Nakase

1. AJ 4792 2. FERM-P 889 3. T. Nakase: J. Gen. Appl. Microbiol., 17, 409 (1971), in English, with Latin description.

Candida fimetaria Soneda

1. SM 2-11 2. NI 7631 3. M. Soneda: Nagaoa, 6, 1 (1959), in English.

4. Candida lambica (Lindner et Genoud) van Uden et Buckley.

Candida fimetaria Soneda var. diversa Ohara, Nonomura et Yunome

1. K-14 2. IFO 1091 3. U. Ohara, H. Nonomura and H. Yunome: J. Agr.

Chem. Soc. Japan, 34, 709 (1960), in Japanese. 4. Candida diversa Ohara,

Nonomura et Yunome ex van Uden et Buckley.

Candida fragicola Nakase

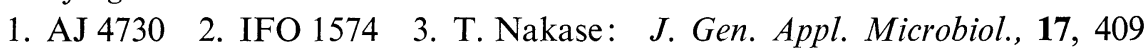

(1971), in English, with Latin description.

Candida fukuoka Sato

3. M. Sato: Fukuoka Igaku Zasshi, 43, 1013 (1952), in Japanese.

Candida glaebosa Nakase et Komagata

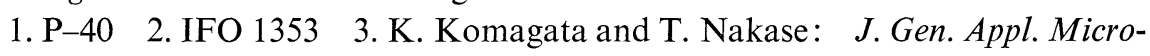

biol., 11, 255 (1965), in English, with Latin description.

Candida guilliermondii (Cast.) Langeron et Guerra var. japonica Sugiyama et Goto

1. KC 1-1 2. RIFY KC 1-1 3. S. Sugiyama and S. Goto: J. Fac. Sci.

Univ. of Tokyo, Sec. III, 10 part 7, 97 (1969), in English, with Latin description.

Candida hydrocarbofumarica Yamada, Furukawa et Nakahara

1. Et-15-2 3. K. Yamada, T. Furukawa and T. Nakahara: Agr. Biol.

Chem., 34, 670 (1970), in English.

Candida incommunis Ohara, Nonomura et Yamazaki

1. K-53 2. RIFY k 53 3. Y. Ohara, H. Nonomura and T. Yamazaki:

J. Gen. Appl. Microbiol., 11, 273 (1965), in English, with Latin description.

Candida ishiwadae Sugiyama et Goto

1. FC 4-1 2. RIFY FC 4-1 3. J. Sugiyama and S. Goto: J. Fac. Sci.

Univ. of Tokyo, Sec. III, 10 part 7, 97 (1969), in English, with Latin description.

Candida koshuensis Yokotsuka et Goto

1. WF 87 2. RIFY WF 87 3. I. Yokotsuka and S. Goto: J. Agr. Chem.

Soc. Japan, 29, 132 (1953), in Japanese.

Candida krusei (Cast.) Berkhout var. komabaensis Kobayashi 
1. No. 72 3. T. Kobayashi: Wood Saccharification Discus. Commit., No. 2, 1 (1953), in English.

Candida lactis Ooki

3. T. Ooki: J. Agr. Chem. Soc. Japan, 33, 1102 (1959), in Japanese.

Candida maltosa Komagata, Nakase et Katsuya

1. Y-2-3 2. CBS 5611 3. K. Komagata et al.: J. Gen. Appl. Microbiol.,

10, 323 (1964), in English, with Latin description. 4. Candida sake (Saito et

Ota) van Uden et Buckley.

Candida oleophila Iizuka

$\begin{array}{llll}\text { 1. ATCC } 20177 & \text { 2. ATCC } 20177 & \text { 3. J. Simizu, K. Ishii, Y. Nakajima and }\end{array}$

H. Iizuka: Japanese Patent 70-39035.

Candida paralipolytica Yamada et Ota

1. No. 8 3. K. Yamada and Y. Ota: J. Agr. Chem. Soc. Japan, 37, 649 (1963), in Japanese.

Candida parapsilosis (Ashf.) Langeron et Talice var. hokkai Goto et Yokotsuka

1. H 32 2. IAM 4488 3. S. Goto and I. Yokotsuka: Bull. Res. Inst. Ferment. Yamanashi Univ., 9, 79 (1962), in Japanese.

Candida parapsilosis (Ashf.) Langeron et Talice var. japonica Kobayashi

1. No. 87 3. T. Kobayashi: Wood Saccharification Discus. Commit., 2, 1 (1953), in English.

Candida parapsilosis (Ashf.) Langeron et Talice var. komabaensis Kobayashi

1. No. 75 3. T. Kobayashi: Wood Saccharification Discus. Commit., 2, 1 (1953), in English.

Candida parapsilosis (Ashf.) Langeron et Talice var. tokyoensis Kobayashi

1. No. 78 3. T. Kobayashi: Wood Saccharification Discus. Commit., 2, 1 (1953), in English.

Candida petrophilum Iguchi, Takeda et Senoo

1. 144 2. ATCC 20226 3. T. Iguchi, I. Takeda and S. Senoo: Japanese Patent 65-20666 (1965), in Japanese.

Candida polymorpha Ohara et Nonomura

1. S-3 2. IFO 0836 3. Y. Ohara and H. Nonomura: J. Agr. Chem. Soc.

Japan, 28, 717 (1964), in Japanese. 4. Candida diddensii (Phaff, Mrak et Williams) Fell et Meyer.

Candida polymorpha Ohara et Nonomura var. shiokara Zenitani

3. B. Zenitani: Sci. Bull. Fac. Agr. Kyushu Univ., 15, 353 (1955), in English.

Candida punicea Komagata et Nakase

1. P-12. 2. IFO 1337 3. K. Komagata and T. Nakase: J. Gen. Appl. Microbiol., 11, 255 (1965), in English, with Latin description.

Candida quercuum Nakase

1. AJ 4781 2. IFO 1576 3. T. Nakase: J. Gen. Appl. Microbiol., 17, 469 (1971), in English, with Latin description.

Candida rugopelliculosa Nakase 
$\begin{array}{lll}\text { 1. AJ } 4656 & \text { 2. IFO } 1577 & \text { 3. T. Nakase: J. Gen. Appl. Microbiol., 17, } 383\end{array}$ (1971), in English, with Latin description.

Candida salmonicola Komagata et Nakase

1. P-32 2. IFO 1354 3. K. Komagata and T. Nakase: J. Gen. Appl.

Microbiol., 11, 255 (1965), in English, with Latin description.

Candida sorboxylosa Nakase

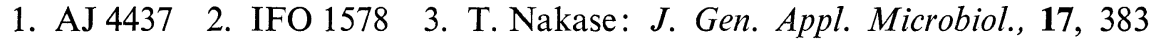

(1971), in English, with Latin description.

Candida talis Sugimori

$\begin{array}{llll}\text { 1. AKU } 4901 & \text { 2. AKU } 4901 & \text { 3. T. Sugimori: Japanese Patent 68-28958 }\end{array}$ (1968), in Japanese.

Candida terebra Sugiyama et Goto

1. KC 1-2 2. RIFY KC 1-2 3. J. Sugiyama and S. Goto: J. Fac. Sci.

Univ. Tokyo, Sec. III, 10 Part 7, 97 (1969), in English, with Latin description.

Candida tropicalis (Cast.) Berkhout var. japonica Kobayashi

3. T. Kobayashi: Wood Saccharification Discus. Commit., 2, 1 (1953), in English.

Candida vinaria Ohara, Nonomura et Yunome

1. O-7 2. IFO 1092 3. Y. Ohara, H. Nonomura and H. Yunome: J. Agr.

Chem. Soc. Japan, 34, 709 (1960), in Japanese. 4. Candida zelanoides (Cast.)

Langeron et Guerra.

Candida yokotsukaensis Yokotsuka et Goto

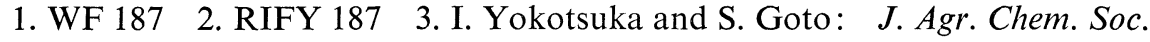

Japan, 29, 132 (1953), in Japanese.

Cryptococcus albidus (Saito) Skinner var. ovalis Sugiyama et Goto

1. K 6-7 2. IFO 1419 3. J. Sugiyama and S. Goto: Jap. J. Bot., 42, 75(1967),

in English, with Latin description. 4. Cryptococcus albidus (Saito) Skinner. Cryptococcus bhutanesis Goto et Sugiyama

1. H 3-9-3 2. RIFY 3-9-3 3. S. Goto and J. Sugiyama: Can. J. Bot., 48, 2097 (1969), in English, with Latin description.

Cryptococcus diffluens (Zach) Lodder var. non-membranaefaciens Ohara et Nonomura

3. Y. Ohara and H. Nonomura: J. Agr. Chem. Soc. Japan, 28, 717 (1954), in Japanese.

Cryptococcus himalayaensis Goto et Sugiyama

1. H 3-11-6 2. RIFY H 3-11-16 3. S. Goto and J. Sugiyama: Can. J. Bot., 48, 2097 (1969), in English, with Latin description.

Debaryomyces Burnieri Ota

3. M. Ota: Ann. Parasitol., 2, 34 (1924), in French.

Debaryomyces emphysematosus Ota

3. M. Ota: Dermatol. Wochenschr., 78, 284 (1924), in German.

Debaryomyces Fabrii Ota 
2. IFO 0015 3. M. Ota: Dermatol. Wochenschr., 78, 284 (1924,) in German.

4. Debaryomyces hansenii (Zopf.) Lodder et Kreger-van Rij.

Debaryomyces franciscae (Capriotti) Kodama, Kyono, Naganishi et Takahara

2. IFO 1360 3. K. Kodama, T. Kyono, H. Naganishi and Y. Takahara:

J. Ferment. Technol., 42, 1 (1964), in Japanese. 4. Saccharomyces pretoriensis van der Walt et Tschenschner.

Debaryomyces fukuyamaensis Naganishi

2. IFO 0037 3. H. Naganishi: Nippon Gakujutsu-kyokai Hokoku, 15, 419

(1941), in Japanese. 4. Debaryomyces hansenii (Zopf.) Lodder et Kreger-van Rij.

Debaryomyces Gruetzii Ota

2. IFO 0017 3. M..Ota: Dermatol. Wochenschr., 78, 284 (1924), in German.

4. Debaryomyces hansenii (Zopf.) Lodder et Kreger-van Rij.

Debaryomyces halotolerans Sasaki et Yoshida

3. Y. Sasaki and T. Yoshida: J. Ferment. Technol., 44, 61 (1966), in Japanese. Debaryomyces Mildegaardi Ota

2. IFO 0047 3. M. Ota: Ann. Parasitol., 1, 124 (1923), in French. 4.

Debaryomyces hansenii (Zopf.) Lodder et Kreger-van Rij.

Debaryomyces japonicus Naganishi

1. D-2 2. IFO 0039 3. H. Naganishi: J. Brew. Sci., 19, 14 (1941), in Japanese. 4. Debaryomyces hansenii (Zopf.) Lodder et Kreger-van Rij.

Debaryomyces Laedegaardi Ota

2. IFO 0042 3. M. Ota: Ann. Parasitol., 1, 124 (1923), in French. 4.

Debaryomyces hansenii (Zopf.) Lodder et Kreger-van Rij.

Debaryomyces Leopoldi Ota

3. M. Ota: Ann. Parasitol., 1, 124 (1923), in French. 4. Debaryomyces hansenii (Zopf.) Lodder et Kreger-van Rij.

Debaryomyces Lundsgaardi Ota

3. M. Ota: Ann. Parasitol., 1, 124 (1923), in French. 4. Debaryomyces hansenii (Zopf.) Lodder et Kreger-van Rij.

Debaryomyces mandshuricus Naganishi

2. IFO 0021 3. H. Naganishi: Rep. Central Lab. S. Manchuria Rail. Co., 10, 425 (1924), in Japanese. 4. Saccharomyces montanus Phaff, Miller et Shifrine.

Debaryomyces membranaefaciens Naganishi

2. IFO 0094 3. N. Naganishi: Rep. Cent. Lab. S. Manchuria Rail. Co.,

4, 187 (1918), in Japanese. 4. Debaryomyces hansenii(Zopf.) Lodder et Kregervan $\mathrm{Rij}$.

Debaryomyces membranaefaciens Naganishi var. zingiberi Otani

3. Y. Otani: J. Brew. Sci., 10, 186 (1932), in Japanese.

Debaryomyces miso Mogi

1. H 12 2. IFO 0057 3. M. Mogi: J. Agr. Chem. Soc. Japan, 14, 1297 
(1938), in Japanese. 4. Debaryomyces hansenii (Zopf.) Lodder et Kreger-van Rij. Debaryomyces miso Mogi var. 1 Mogi

1. H 22 2. IFO 0098 3. M. Mogi: J. Agr. Chem. Soc. Japan, 14, 1297 (1938), in Japanese.

Debaryomyces nepalensis Goto et Sugiyama

1. Np 2-17-5 2. RIFY 2-17-5 3. S. Goto and J. Sugiyama: Jap. J. Bot., 43, 102 (1968), in English, with Latin description. Debaryomyces nicotianae Naganishi var. salsus Zenitani

3. B. Zenitani: Sci. Bull. Fac. Agr. Kyushu Univ., 14, 306 (1953), in English. Debaryomyces nilssoni (Capriotti) Kodama, Kyono, Naganishi et Takahashi

1. D-637 2. IFO 1255 3. K. Kodama, T. Kyono, H. Naganishi and Y. Takahashi: J. Ferment. Technol., 42, 655 (1964), in Japanese. 4. Saccharomyces microellipsodes Osterwalder.

Debaryomyces orientalis Naganishi

1. D 1 2. IFO 0058 3. H. Naganishi: J. Brew. Sci., 19, 14 (1941), in Japanese. 4. Debaryomyces hansenii (Zopf.) Lodder et Kreger-van Rij.

Debaryomyces sake Saito et Oda

1. KI 4 2. IFO 0060 3. K. Saito and M. Oda: J. Brew. Sci., 10, 787 (1932), in Japanese. 4. Debaryomyces hansenii (Zopf.) Lodder et Kreger-van Rij.

Debaryomyces tamarii Ohara et Nonomura

1. M-12 2. IFO 0854 3. Y. Ohara and H. Nonomura: J. Agr. Chem. Soc. Japan, 28, 337 (1974), in Japanese.

Debaryomyces tremoniensis Ota

3. M. Ota: Dermatol. Woch., 78, 284 (1924), in German. 4. Debaryomyces hansenii (Zopf.) Lodder et Kreger-van Rij.

Endomyces hordei Saito

2. IFO 0104 3. K. Saito: Rep. Cent. Lab. S. Manchuria Rail. Co., 1, separate part No. 1, 1 (1914), in German. 4. Endomycopsis fibuligera (Lindner) Dekker.

Endomyces lindneri Saito

2. IFO 0106 3. K. Saito: Zeit. Gärungsphys., 2, 151 (1913), in German.

4. Endomycopsis fibuligera (Lindner) Dekker.

Endomycopsis fukushimae Soneda

3. M. Soneda: Mycol. Soc. Japan, 3, 36 (1962), in English, with Latin description. 4. Hansenula californica (Lodder) Wickerham.

Endomycopsis monosporus Saito

2. IFO 0113 3. K. Saito: J. Brew. Sci., 10, 11 (1932), in Japanese.

Endomycopsis muscicola Nakase et Komagata

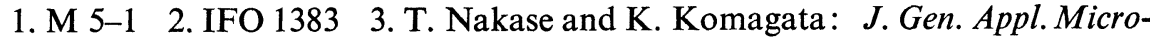
biol., 12, 347 (1966), in English, with Latin description.

Endomycopsis acolyti Phaff et Yoneyama

2. IFO 1113 3. H. J. Phaff and M. Yoneyama: Antonie van Leeuwenhoek, 
27, 196 (1961), in English, with Latin description. 4. Pichia scolyti (Phaff et Yoneyama) Kreger-van Rij.

Eutorulopsis frugi Oda et Nakamori

3. M. Oda and Nakamori: J. Ferment. Technol., 27, 211 (1949), in Japanese. Eutorulopsis Sake Saito et Oda

3. K. Saito and M. Oda: J. Brew. Sci., 12, 160 (1934), in Japanese. 4. Candida sake (Saito et Oda) van Uden et Buckley.

(To be continued to the next issue.) 